\title{
On an eigenvalue problem involving the Hardy potential
}

\author{
J. Chabrowski \\ Department of Mathematics, University of Queensland \\ St. Lucia 4072, Qld, Australia \\ I. Peral \\ Departamento de Matemáticas \\ Universidad Autónoma de Madrid \\ 28049 Madrid Spain \\ B. Ruf \\ Dipartimento de Matematica \\ Universita degli Studi di Milano \\ Via Saldini 50, I-20133 Milano Italy
}

\begin{abstract}
In this note we consider the eigenvalue problem for the Laplacian with the Neumann and Robin boundary conditions involving the Hardy potential. We prove the existence of eigenfunctions of the second eigenvalue for the Neumann problem and of the principal eigenvalue for the Robin problem in "high" dimensions.
\end{abstract}

\section{Introduction}

In this note we assume that $\Omega \subset \mathbb{R}^{N}, N \geq 3$, is a bounded, connected open set with a smooth boundary $\partial \Omega$. We consider the following eigenvalue problem

$$
\begin{cases}-\Delta u & =\mu \frac{1}{|x|^{2}} u \text { in } \Omega, \\ \frac{\partial u}{\partial \nu} & =0 \text { on } \partial \Omega .\end{cases}
$$

2000 Mathematics Subject Classification: 35J20, 35P15, 49R50.

Key words and phrases: Poincaré inequality, Hardy potential, eigenvalue problems 
Solutions to this problem are sought in the Sobolev space $W^{1,2}(\Omega)$. We recall that $W^{1,2}(\Omega)$ is the Sobolev space equipped with norm

$$
\|u\|_{W^{1,2}}^{2}=\int_{\Omega}\left(|\nabla u|^{2}+u^{2}\right) d x .
$$

$W_{\circ}^{1,2}(\Omega)$ is a subspace of $W^{1,2}(\Omega)$ consisting of functions vanishing in the sense of trace on the boundary $\partial \Omega$ of $\Omega$. The norm $\|u\|_{W^{1,2}}$ on this subspace is equivalent to

$$
\|u\|_{W_{\circ}^{1,2}}^{2}=\int_{\Omega}|\nabla u|^{2} d x
$$

Problem (1.1) is closely related to the Hardy inequality. The Hardy inequality in $W_{\circ}^{1,2}(\Omega)$ has the following form: let $0 \in \Omega$, then

$$
\int_{\Omega} \frac{u^{2}}{|x|^{2}} d x \leq \frac{1}{\Lambda_{N}} \int_{\Omega}|\nabla u|^{2} d x
$$

for every $u \in W_{\circ}^{1,2}(\Omega)$, where $\Lambda_{N}=\frac{(N-2)^{2}}{4}$. The constant $\Lambda_{N}$ is an optimal constant and there is no nonzero function in $W_{\circ}^{1,2}(\Omega)$ which changes this inequality into equality (see [10], Theorem 4.1). In recent years the Hardy inequality in $W_{\circ}^{1,2}(\Omega)$ and $W^{1,2}(\Omega)$ and the related problems for semilinear elliptic equation have attracted considerable interest. We refer to papers [6] and [7], where further bibliographical references can be found. For further generalizations and applications of the Hardy - Sobolev type inequalities, we refer to the papers [1], [2], [3], [4], [16]. The value of the minimum and its attainability of the Rayleigh with respect to the boundary conditions has been investigated in [5]. According to the results of this paper the optimal constant in $W^{1,2}(\Omega)$ is zero and is attained by constant functions. Thus to obtain an extension of $(1.2)$ to $W^{1,2}(\Omega)$ involving the whole norm, it is necessary to add a new positive term to the right hand side of (1.2). This question is discussed in the papers [6] and [7]. In this note we need the following extension of $(1.2)$ to the space $W^{1,2}(\Omega)$.

Proposition 1.1 Let $0 \in \bar{\Omega}$. Then for every $\delta>0$ there exists a constant $A=A(\delta, \Omega)$ such that

$$
\int_{\Omega} \frac{u^{2}}{|x|^{2}} d x \leq\left(\frac{1}{\Lambda_{N}}+\delta\right) \int_{\Omega}|\nabla u|^{2} d x+A \int_{\Omega} u^{2} d x
$$

for every $u \in W^{1,2}(\Omega)$.

The proof of (1.3) can be found in papers [13] and [14]. The case $0 \in \Omega$ is considered in [14] and the case $0 \in \partial \Omega$ in [13] (see also [19]).

The paper is organized as follows. In Section 2 we discuss the inequalities of Hardy Poincaré type. These inequalities lead to the eigenvalue problem (1.1). A sufficient condition for the existence of the second eigenvalue and the corresponding eigenfunction is presented in Section 3, namely that the corresponding Rayleigh quotient lies strictly below the Hardy constant $\Lambda_{N}$ (see (3.1)). In Section 4 we give examples of sets which satisfy this condition 
provided the dimension is sufficiently large. The eigenfunctions we find may be singular at the origin. Section 5 is devoted to the study of the asymptotic behavior of the second eigenfunction of (1.1) around 0 . Since $\Lambda_{N}$ is not attained on the space $W_{\circ}^{1,2}(\Omega)$ there is no analogue to problem (1.1) with Dirichlet boundary conditions. In Section 6 we briefly discuss the eigenvalue problem involving the Hardy potential with Robin boundary conditions. We show that the smallest eigenvalue for this problem is attained if it lies below the Hardy constant $\Lambda_{N}$; again, this is the case if the space-dimension is sufficiently large. In the final Section 7 we formulate some open problems related to this paper.

Problems (1.1) and (6.1) investigated in this paper are eigenvalue problems with weight functions. The weight function $\frac{1}{|x|^{2}}$ considered here is not in $L^{\frac{N}{2}}(\Omega) \cap L^{\infty}(\Omega)$ and consequently the functional $u \rightarrow \int_{\Omega} \frac{u^{2}}{|x|^{2}} d x$ is not completely continuous in $W^{1,2}(\Omega)$ unlike in papers [8], [11] and [25]. For a good survey of eigenvalue problems for the $p$-Laplacian, however without the Hardy potential, we refer to paper [20].

Throughout this paper in a given Banach space $X$ we denote strong convergence by " $\rightarrow$ " and weak convergence by " $\rightarrow$ ". The norms in the Lebesgue spaces $L^{p}(\Omega), 1<p<\infty$, are denoted by $\|\cdot\|_{p}$.

\section{The inequality of the Hardy - Poincaré type}

We denote by $L^{2}\left(\Omega, \frac{1}{|x|^{2}} d x\right)$ the weighted Lebesgue space equipped with norm

$$
\|u\|_{*}^{2}=\int_{\Omega} \frac{u^{2}}{|x|^{2}} d x .
$$

The corresponding scalar product on $L^{2}\left(\Omega, \frac{1}{|x|^{2}}\right)$ is denoted by $(\cdot, \cdot)_{*}$.

Theorem 2.1 Suppose that $0 \in \bar{\Omega}$ and that $g \in L^{2}\left(\Omega, \frac{1}{|x|^{2}}\right)$ with $\int_{\Omega} \frac{g}{|x|^{2}} d x=1$. We assume that $\Omega$ is an open, connected and bounded set with a smooth boundary. Then there exists a constant $H=H(g, \Omega)$ such that

$$
\int_{\Omega}\left(u-\int_{\Omega} \frac{u g}{|x|^{2}} d x\right)^{2} \frac{d x}{|x|^{2}} \leq H \int_{\Omega}|\nabla u|^{2} d x
$$

for every $u \in W^{1,2}(\Omega)$.

The proof is similar to that of Theorem 8.11 in [21] and is omitted. We list below some consequences of Theorem 2.1.

Corollary 2.2 We make now specific choices of $g$. 
(a) If $g=\frac{1}{\int_{\Omega} \frac{d x}{|x|^{2}}}$, then (2.1) takes the form: there exists a constant $H>0$ such that

$$
\int_{\Omega}\left(u-\frac{1}{\int_{\Omega} \frac{d x}{|x|^{2}}} \int_{\Omega} \frac{u}{|x|^{2}} d x\right)^{2} \frac{d x}{|x|^{2}} \leq H \int_{\Omega}|\nabla u|^{2} d x
$$

for every $u \in W^{1,2}(\Omega)$.

(b) If $g=\frac{|x|^{2}}{|\Omega|}$, then (2.1) takes the form: there exists a constant $H>0$ such that

$$
\int_{\Omega}\left(u-\frac{1}{|\Omega|} \int_{\Omega} u d x\right)^{2} \frac{d x}{|x|^{2}} \leq H \int_{\Omega}|\nabla u|^{2} d x
$$

for every $u \in W^{1,2}(\Omega)$.

(c) If $g=|x|^{2} h$ with $h \in L^{2}(\Omega)$ and $\int_{\Omega} h d x=1$, then (2.1) takes the form: there exists a constant $H>0$ such that

$$
\int_{\Omega}\left(u-\int_{\Omega} u h d x\right)^{2} \frac{d x}{|x|^{2}} \leq H \int_{\Omega}|\nabla u|^{2} d x
$$

for every $u \in W^{1,2}(\Omega)$.

From the case $(c)$ we derive the following inequality

$$
\frac{1}{d^{2}} \int_{\Omega}\left(u-\int_{\Omega} u h d x\right)^{2} d x \leq \int_{\Omega}\left(u-\int_{\Omega} u h d x\right)^{2} \frac{d x}{|x|^{2}} \leq H \int_{\Omega}|\nabla u|^{2} d x,
$$

where $d=\operatorname{diam} \Omega$.

If we restrict $(2.1)$ to the subspace of $W^{1,2}(\Omega)$ consisting of functions orthogonal to $g$ in $L^{2}\left(\Omega, \frac{1}{|x|^{2}} d x\right)$, then $(2.1)$ is reduced to the following inequality: given $g \in L^{2}\left(\Omega, \frac{1}{|x|^{2}}\right)$ with $\int_{\Omega} \frac{g}{|x|^{2}} d x=1$, then there exists a constant $H>0$ such that

$$
\int_{\Omega} \frac{u^{2}}{|x|^{2}} d x \leq H \int_{\Omega}|\nabla u|^{2} d x
$$

for every $u \in W^{1,2}(\Omega)$ with $(u, g)_{*}=0$. This observation can be applied to the cases $(a),(b)$ and $(c)$ of Corollary 2.2. In particular, from the case $(a)$ we obtain the following inequality: there exists a constant $H>0$ such that

$$
\int_{\Omega} \frac{u^{2}}{|x|^{2}} d x \leq H \int_{\Omega}|\nabla u|^{2} d x
$$

for every $u \in W^{1,2}(\Omega)$ with $\int_{\Omega} \frac{u}{|x|^{2}} d x=0$. The reciprocal of the smallest constant $H$ satisfying inequality (2.2) is the second eigenvalue of the eigenvalue problem (1.1). The first eigenvalue $\lambda_{1}=0$ and the corresponding eigenfunctions are constant. 
Using the the triangle inequality we deduce the following estimate from (2.1): given $g \in L^{2}\left(\Omega, \frac{1}{|x|^{2}}\right)$ with $\int_{\Omega} \frac{g}{|x|^{2}} d x=1$, there exists a constant $H>0$ such that

$$
\left(\int_{\Omega} \frac{u^{2}}{|x|^{2}} d x\right)^{\frac{1}{2}} \leq H^{\frac{1}{2}}\left(\int_{\Omega}|\nabla u|^{2} d x\right)^{\frac{1}{2}}+\left(\int_{\Omega} \frac{d x}{|x|^{2}}\right)^{\frac{1}{2}}\left|\int_{\Omega} \frac{u g}{|x|^{2}} d x\right|
$$

for every $u \in W^{1,2}(\Omega)$. In the case $(a)$ of Corollary 2.2 this inequality takes the form: there exists a constant $H>0$ such that

$$
\left(\int_{\Omega} \frac{u^{2}}{|x|^{2}} d x\right)^{\frac{1}{2}} \leq H^{\frac{1}{2}}\left(\int_{\Omega}|\nabla u|^{2} d x\right)^{\frac{1}{2}}+\left(\int_{\Omega} \frac{d x}{|x|^{2}}\right)^{-\frac{1}{2}}\left|\int_{\Omega} \frac{u}{|x|^{2}} d x\right|
$$

for every $u \in W^{1,2}(\Omega)$. Obviously, similar inequalities can be established in the cases $(b)$ and $(c)$.

\section{The second eigenvalue}

The second eigenvalue $\lambda_{2}^{H}$ of the eigenvalue problem (1.1) is defined by

$$
\lambda_{2}^{H}=\inf _{u \in W^{1,2}(\Omega)-\{0\}, \int_{\Omega} \frac{u}{|x|^{2}} d x=0} \frac{\int_{\Omega}|\nabla u|^{2} d x}{\int_{\Omega} \frac{u^{2}}{|x|^{2}} d x} .
$$

Theorem 3.1 Suppose that

$$
\lambda_{2}^{H}<\Lambda_{N}
$$

Then there exists an eigenfunction $\varphi_{2}$ corresponding to the eigenvalue $\lambda_{2}^{H}$.

Proof Let $\left\{u_{n}\right\}$ be a minimizing sequence for $\lambda_{2}^{H}: \int_{\Omega}\left|\nabla u_{n}\right|^{2} d x \rightarrow \lambda_{2}^{H}$ with $\int_{\Omega} \frac{u_{n}}{|x|^{2}} d x=0$ and $\int_{\Omega} \frac{u_{n}^{2}}{|x|^{2}} d x=1$ for each $n$. We may assume that $u_{n} \rightarrow u$ in $W^{1,2}(\Omega)$ and $L^{2}\left(\Omega, \frac{d x}{|x|^{2}}\right)$ and $u_{n} \rightarrow u$ a.e. on $\Omega$. By the P.L. Lions' concentration - compactness principle [23] there exist constants $\mu_{\circ}, \nu_{\circ} \geq 0$ such that

$$
\begin{gathered}
\left|\nabla u_{n}\right|^{2} \rightarrow \mu \geq|\nabla u|^{2}+\mu_{\circ} \delta_{\circ}, \\
\frac{u_{n}^{2}}{|x|^{2}} \rightarrow \nu=\frac{u^{2}}{|x|^{2}}+\nu_{\circ} \delta_{\circ}
\end{gathered}
$$

in the sense of measures and

$$
\nu_{\circ} \Lambda_{N} \leq \mu_{\circ}
$$


where $\delta_{\circ}$ is the Dirac measure assigned to 0 . First, we show that $u \not \equiv 0$. Arguing by contradiction assume that $u \equiv 0$ on $\Omega$. Then $\nu_{\circ}=1$ and $\Lambda_{N} \leq \mu_{\circ}$. From (3.2) we derive that

$$
\Lambda_{N} \leq \mu_{\circ} \leq \lambda_{2}^{H}<\Lambda_{N}
$$

which is impossible. If $\nu_{\circ}=0$ we are done. So it remains to consider the case $0<\nu_{\circ}$. In this case we have

$$
\begin{aligned}
\lambda_{2}^{H} & \geq \int_{\Omega}|\nabla u|^{2} d x+\mu_{\circ} \geq \int_{\Omega}|\nabla u|^{2} d x+\nu_{\circ} \Lambda_{N} \\
& \geq \int_{\Omega}|\nabla u|^{2} d x+\Lambda_{N}\left(1-\int_{\Omega} \frac{u^{2}}{|x|^{2}} d x\right) .
\end{aligned}
$$

This yields

$$
\lambda_{2}^{H}-\Lambda_{N} \geq \int_{\Omega}|\nabla u|^{2} d x-\Lambda_{N} \int_{\Omega} \frac{u^{2}}{|x|^{2}} d x .
$$

Since $\int_{\Omega} \frac{u}{|x|^{2}} d x=0, u$ cannot be a constant function. Hence $\int_{\Omega}|\nabla u|^{2} d x>0$. From (3.5) we deduce that

$$
\Lambda_{N}+\frac{\lambda_{2}^{H}-\Lambda_{N}}{\int_{\Omega} \frac{u^{2}}{|x|^{2}} d x} \geq \frac{\int_{\Omega}|\nabla u|^{2} d x}{\int_{\Omega} \frac{u^{2}}{|x|^{2}} d x} \geq \lambda_{2}^{H} .
$$

Hence

$$
\Lambda_{N}-\lambda_{2}^{H} \geq \frac{\Lambda_{N}-\lambda_{2}^{H}}{\int_{\Omega} \frac{u^{2}}{|x|^{2}} d x} .
$$

This implies that $\int_{\Omega} \frac{u^{2}}{|x|^{2}} d x \geq 1$ which is impossible because $\int_{\Omega} \frac{u^{2}}{|x|^{2}} d x<1$. To complete the proof we show that $u$ is a weak solution to problem (1.2). Let $\phi \in W^{1,2}(\Omega)$ with $\int_{\Omega} \frac{\phi}{|x|^{2}} d x=0$. We put

$$
h(t)=\frac{\int_{\Omega}|\nabla(u+t \phi)|^{2} d x}{\int_{\Omega} \frac{(u+t \phi)^{2}}{|x|^{2}} d x}
$$

for $t \in \mathbb{R}$. Since the function $h$ attains a minimum at $t=0$ we get

$$
\int_{\Omega} \nabla u \nabla \phi d x-\lambda_{2}^{H} \int_{\Omega} \frac{u \phi}{|x|^{2}} d x=0
$$

To extend this identity for arbitrary $\phi \in W^{1,2}(\Omega)$ we set $\psi=\phi-\frac{\int_{\Omega} \frac{\phi}{|x|^{2}} d x}{\int_{\Omega} \frac{1}{|x|^{2}} d x}$. Then $\int_{\Omega} \frac{\psi}{|x|^{2}} d x=0$ and

$$
\int_{\Omega} \nabla u \nabla \psi d x=\int_{\Omega} \nabla u \nabla \phi d x=\lambda_{2}^{H} \int_{\Omega} \frac{u \psi}{|x|^{2}} d x=\lambda_{2}^{H} \int_{\Omega} \frac{u \phi}{|x|^{2}} d x
$$

since $u$ is orthogonal to 1 in $L^{2}\left(\Omega, \frac{d x}{|x|^{2}}\right)$. 
Remark 3.2 If $0<\mu<\lambda_{2}^{H}$, then problem (1.1) has only the trivial solution in $W^{1,2}(\Omega)$. Indeed, suppose that $u \not \equiv 0$ belonging to $W^{1,2}(\Omega)$ satisfies (1.1). Then $\int_{\Omega} \frac{u}{|x|^{2}} d x=0$ and

$$
\frac{\int_{\Omega}|\nabla v|^{2} d x}{\int_{\Omega} \frac{v^{2}}{|x|^{2}}}=\mu<\lambda_{2}^{H}
$$

which is impossible.

\section{$4 \quad$ Examples of sets satisfying (3.1)}

In this section we give examples of domains satisfying (3.1). These domains have some sort of symmetry or satisfy a pinching condition, and they are rather high dimensional.

1. Let $\Omega=B(0, R)$. First of all we observe that there are two radial solutions of equation (1.1) for $0<\lambda<\Lambda_{N}$ :

$$
u_{1}=r^{-\frac{N-2}{2}+\sqrt{\left(\frac{N-2}{2}\right)^{2}-\lambda}} \text { and } u_{2}=r^{-\frac{N-2}{2}-\sqrt{\left(\frac{N-2}{2}\right)^{2}-\lambda}} .
$$

Since $u_{2} \notin W^{1,2}(B(0, R))$ we cannot use these radial functions to construct a solution of (1.1) for $\lambda=\lambda_{2}^{H}$. To verify (3.1) one can use the coordinate function $u=x_{j}$. It is easy to show that $\lambda_{2}^{H} \leq N$. Hence (3.1) is satisfied for $N \geq 8$. This can be slightly improved by taking as a test function $v(x)=\frac{x_{N}}{|x|}$. We then have

$$
\begin{aligned}
\int_{B(0, R)}|\nabla v(x)|^{2} d x & =\int_{B(0, R)}\left(\frac{1}{|x|^{2}}-\frac{x_{N}^{2}}{|x|^{4}}\right) d x=\left(1-\frac{1}{N}\right) \int_{B(0, R)} \frac{1}{|x|^{2}} d x \\
& =\omega_{N-1} R^{N-2} \frac{N-1}{N(N-2)}
\end{aligned}
$$

Similarly, we have

$$
\int_{B(0, R)} \frac{v(x)^{2}}{|x|^{2}} d x=\int_{B(0, R)} \frac{x_{N}^{2}}{|x|^{4}} d x=\frac{1}{N} \int_{B(0, R)} \frac{1}{|x|^{2}} d x=\omega_{N-1} \frac{R^{N-2}}{N(N-2)} .
$$

Hence $\lambda_{2}^{H} \leq N-1$ and (3.1) is satisfied for $N \geq 7$.

2. This observation can be extended to sets which are close to balls, i.e. satisfying a pinching condition. Let $\Omega$ be a bounded domain in $\mathbb{R}^{N}$. Suppose that there exists a ball $B(0, R) \subset \Omega$ satisfying the following condition

$$
(*) \quad|\Omega|<\frac{1}{N}\left(\frac{N-2}{2}\right)^{2}|B(0, R)| .
$$

Then (3.1) holds. This condition is meaningful if $\frac{1}{N}\left(\frac{N-2}{2}\right)^{2}>1$, that is $N \geq 8$. Obviously we can assume that $B(0, R)$ is largest ball contained in $\Omega$ and satisfying $\left(^{*}\right)$. We distinguish 
two cases: (i) $\int_{\Omega} \frac{x_{j}}{|x|^{2}} d x=0$ for some $1 \leq j \leq N$ and (ii) $\int_{\Omega} \frac{x_{j}}{|x|^{2}} d x \neq 0$ for every $j=1, \ldots, N$. In the first case take $u=x_{j}$; then $\int_{\Omega}\left|\nabla\left(x_{j}\right)\right|^{2} d x=|\Omega|$ and

$$
\int_{\Omega} \frac{x_{j}^{2}}{|x|^{2}} d x \geq \int_{B(0, R)} \frac{x_{j}^{2}}{|x|^{2}} d x=\frac{1}{N}|B(0, R)| .
$$

Hence $\lambda_{2}^{H} \leq \frac{N|\Omega|}{|B(0, R)|}$. If (ii) holds we put $u(x)=\alpha_{k} x_{j}-\alpha_{j} x_{k}$, where $\alpha_{j}=\int_{\Omega} \frac{x_{j}}{|x|^{2}} d x$ and $\alpha_{k}=\int_{\Omega} \frac{x_{k}}{|x|^{2}} d x$. It is clear that $\int_{\Omega} \frac{u}{|x|^{2}} d x=0$. We also have $\int_{\Omega}|\nabla u|^{2} d x=\left(\alpha_{k}^{2}+\alpha_{j}^{2}\right)|\Omega|$ and

$$
\begin{aligned}
\int_{\Omega} \frac{u^{2}}{|x|^{2}} d x & \geq \int_{B(0, R)} \frac{\alpha_{k}^{2} x_{j}^{2}+\alpha_{j}^{2} x_{k}^{2}-2 \alpha_{j} \alpha_{k} x_{j} x_{k}}{|x|^{2}} d x \\
& =\frac{1}{N}\left(\alpha_{k}^{2}+\alpha_{j}^{2}\right)|B(0, R)| .
\end{aligned}
$$

Again we obtain the estimate $\lambda_{2}^{H} \leq \frac{N|\Omega|}{|B(0, R)|}$. Since $(*)$ holds we get in both cases condition (3.1).

3. We now consider sets having a symmetry with respect to the coordinate $x_{N}$. Set $x=\left(x^{\prime}, x_{N}\right)$ and let $\Omega=\left\{x^{\prime} \in D ;-f\left(x^{\prime}\right)<x_{N}<f\left(x^{\prime}\right)\right\}$, where $f: \bar{D} \rightarrow(0, \infty)$ is a smooth function satisfying

$$
\int_{D} f\left(x^{\prime}\right)^{3} d x^{\prime}>\frac{(12)^{\frac{3}{2}} d^{3}|D|}{(N-2)^{3}} .
$$

We show that (3.1) is satisfied. Take $u=x_{N}$; it is clear that $\int_{\Omega} \frac{x_{N}}{|x|^{2}} d x=0$. We have

$$
\int_{\Omega}\left|\nabla\left(x_{N}\right)\right|^{2} d x=\int_{D} d x^{\prime} \int_{-f\left(x^{\prime}\right)}^{f\left(x^{\prime}\right)} d x_{N}=2 \int_{D} f\left(x^{\prime}\right) d x^{\prime}
$$

and

$$
\int_{\Omega} \frac{x_{N}^{2}}{|x|^{2}} d x \geq \frac{1}{d^{2}} \int_{\Omega} x_{N}^{2} d x=\frac{2}{3 d^{2}} \int_{D} f\left(x^{\prime}\right)^{3} d x^{\prime}
$$

Hence with the aid of the Hölder inequality and $(* *)$ we obtain

$$
\lambda_{2}^{H} \leq \frac{\int_{\Omega}\left|\nabla\left(x_{N}\right)\right|^{2} d x^{\prime}}{\int_{\Omega} \frac{x_{N}^{2}}{|x|^{2}} d x^{\prime}} \leq \frac{3 d^{2} \int_{D} f\left(x^{\prime}\right) d x^{\prime}}{\int_{D} f\left(x^{\prime}\right)^{3} d x^{\prime}} \leq 3 d^{2}|D|^{\frac{2}{3}}\left(\int_{D} f\left(x^{\prime}\right)^{3} d x^{\prime}\right)^{-\frac{2}{3}}<\Lambda_{N} .
$$

Let $\operatorname{diam} D \leq d,(d=\operatorname{diam} \Omega)$. Since $\max _{x^{\prime} \in D} f\left(x^{\prime}\right) \leq \frac{d}{2}$ we have

$$
\int_{D} f\left(x^{\prime}\right)^{3} d x^{\prime} \leq\left(\frac{d}{2}\right)^{3}|D|
$$

Thus condition $(* *)$ is meaningful if

$$
\left(\frac{d}{2}\right)^{3}|D|>\frac{(12)^{\frac{3}{2}} d^{3}|D|}{(N-2)^{3}}
$$


that is $N>2+2 \sqrt{12},(N \geq 9)$.

4. Suppose that $\Omega^{\prime} \subset \Omega$, with $\Omega^{\prime}$ as in example 3, that is, $\Omega^{\prime}=\left\{x^{\prime} \in D ;-f\left(x^{\prime}\right)<x_{N}<\right.$ $\left.f\left(x^{\prime}\right)\right\}$, where $f: \bar{D} \rightarrow(0, \infty)$ is a smooth function. Assume that

$$
(* * *) \quad|\Omega|<\frac{2}{3 d_{1}^{2}}\left(\frac{N-2}{2}\right)^{2} \int_{D} f\left(x^{\prime}\right)^{3} d x^{\prime}
$$

where $d_{1}=\operatorname{diam} \Omega^{\prime}$. Then (3.1) holds. To show this we distinguish two cases:

(i) $\int_{\Omega} \frac{x_{N}}{|x|^{2}} d x=0$ and (ii) $\int_{\Omega} \frac{x_{N}}{|x|^{2}} d x \neq 0$. Assuming (i), we choose $u=x_{N}$; then $\int_{\Omega}\left|\nabla\left(x_{N}\right)\right|^{2} d x=$ $|\Omega|$ and

$$
\int_{\Omega} \frac{x_{N}^{2}}{|x|^{2}} d x \geq \frac{1}{d_{1}^{2}} \int_{\Omega^{\prime}} x_{N}^{2} d x=\frac{2}{3 d_{1}^{2}} \int_{D} f\left(x^{\prime}\right)^{3} d x^{\prime}
$$

Condition (3.1) follows from (***). In the case (ii) we test $\lambda_{2}^{H}$ with

$$
u(x)=x_{N}-\frac{\int_{\Omega} \frac{x_{N}}{|x|^{2}} d x}{\int_{\Omega} \frac{d x}{|x|^{2}}} .
$$

It is clear that $\int_{\Omega} \frac{u}{|x|^{2}} d x=0$ and $\int_{\Omega}|\nabla u|^{2} d x=|\Omega|$. Letting $a=\frac{\int_{\Omega} \frac{x_{N}}{|x|^{2}} d x}{\int_{\Omega} \frac{d x}{|x|^{2}}}$ we get

$$
\begin{aligned}
\int_{\Omega} \frac{u^{2}}{|x|^{2}} d x & \geq \int_{\Omega^{\prime}} \frac{u^{2}}{|x|^{2}} d x=\int_{\Omega^{\prime}} \frac{x_{N}^{2}}{|x|^{2}} d x+a^{2} \int_{\Omega^{\prime}} \frac{d x}{|x|^{2}}-2 a \int_{\Omega^{\prime}} \frac{x_{N}}{|x|^{2}} d x \\
& =\int_{\Omega^{\prime}} \frac{x_{N}^{2}}{|x|^{2}} d x+a^{2} \int_{\Omega^{\prime}} \frac{d x}{|x|^{2}} \\
& \geq \frac{2}{3 d_{1}^{2}} \int_{D} f\left(x^{\prime}\right)^{3} d x^{\prime}
\end{aligned}
$$

The estimate (3.1) follows from $(* * *)$.

5. Let $\Omega=\left\{x ;\left(x_{1}-R\right)^{2}+x_{2}^{2}+\ldots+x_{N}^{2} \leq R^{2}\right\}$. In this example $0 \in \partial \Omega$. We test $\lambda_{2}^{H}$ with $u(x)=x_{j}$ for some $2 \leq j \leq N$. We have $\int_{\Omega} \frac{x_{j}}{|x|^{2}} d x=0, \int_{\Omega}\left|\nabla\left(x_{j}\right)\right|^{2} d x=|\Omega|$ and

$$
\int_{\Omega} \frac{x_{j}^{2}}{|x|^{2}} d x \geq \frac{1}{4 R^{2}} \int_{\Omega} x_{j}^{2} d x=\frac{1}{4 R^{2}} \int_{B(0, R)} x_{j}^{2} d x=\frac{1}{4 N R^{2}} \int_{B(0, R)}|x|^{2} d x=\frac{\omega_{N-1} R^{N}}{4 N(N+2)}
$$

Thus

$$
\lambda_{2}^{H} \leq \frac{4 N(N+2)|\Omega|}{\omega_{N-1} R^{N}}=4(N+2)
$$

and (3.1) holds provided $4(N+2)<\left(\frac{N-2}{2}\right)$, that is, $N \geq 22$.

6. We now give an example of a domain satisfying (3.1) which does not have a symmetry with respect to 0 . Let $\Omega$ be a domain from example 3 . We put

$$
\Omega_{\delta}=\left\{x ; x^{\prime} \in D,-f\left(x^{\prime}\right)<x_{N}<(1+\delta) f\left(x^{\prime}\right)\right\},
$$


where $\delta>0$. Let $0<m=\min _{x^{\prime} \in \bar{D}} f\left(x^{\prime}\right)$ and $M=\max _{x^{\prime} \in \bar{D}} f\left(x^{\prime}\right)$. Suppose that diam $D \leq$ $2 M$ and that

$$
(* * * *) \quad \frac{m^{3}}{6 M^{3}}\left(\frac{N-2}{2}\right)^{2}>2+\delta .
$$

(This condition makes sense for $N \geq 9$ ). Then condition (3.1) holds. First we observe that $\int_{\Omega_{\delta}} \frac{x_{N}}{|x|^{2}} d x=\int_{\Omega_{\delta}-\Omega} \frac{x_{N}}{|x|^{2}} d x>0$. So we introduce a function

$$
v(x)=x_{N}-\alpha_{\delta}, \quad \alpha_{\delta}=\frac{\int_{\Omega_{\delta}} \frac{x_{N}}{|x|^{2}} d x}{\int_{\Omega_{\delta}} \frac{d x}{|x|^{2}}} .
$$

It is clear that $\int_{\Omega_{\delta}} \frac{v(x)}{|x|^{2}} d x=0$ and

$$
\int_{\Omega_{\delta}}|\nabla v|^{2} d x=\left|\Omega_{\delta}\right|=(2+\delta) \int_{D} f\left(x^{\prime}\right) d x^{\prime} .
$$

Letting $d=\operatorname{diam} \Omega$ we get

$$
\begin{aligned}
\int_{\Omega_{\delta}} \frac{v^{2}}{|x|^{2}} d x & \geq \int_{\Omega} \frac{v^{2}}{|x|^{2}} d x=\int_{\Omega} \frac{x_{N}^{2}+\alpha_{\delta}^{2}-2 \alpha_{\delta} x_{N}}{|x|^{2}} d x=\int_{\Omega} \frac{x_{N}^{2}+\alpha_{\delta}^{2}}{|x|^{2}} d x \\
& \geq \frac{2}{3 d^{2}} \int_{D} f\left(x^{\prime}\right)^{3} d x^{\prime} .
\end{aligned}
$$

Since $d=2 M$ we obtain the following estimate for $\lambda_{2}^{H}$

$$
\begin{aligned}
\lambda_{2}^{H} & \leq \frac{(2+\delta) \int_{D} f\left(x^{\prime}\right) d x^{\prime}}{\frac{2}{3 d^{2}} \int_{D} f\left(x^{\prime}\right)^{3} d x^{\prime}} \\
& \leq \frac{3(2+\delta)}{2}(2 M)^{2} \frac{M}{m^{3}}=6(2+\delta) \frac{M^{3}}{m^{3}} .
\end{aligned}
$$

Condition (3.1) follows from $(* * * *)$.

According to example 1 we have $\lambda_{2}^{H}=O(N-1)$, as $N \rightarrow \infty$, if $\Omega=B(0, R) \subset \mathbb{R}^{N}$. Obviously it is not clear whether this asymptotic behaviour is optimal. Below we give an example of a domain for which $\lambda_{2}^{H}$ remains bounded as $N \rightarrow \infty$.

7. Let $\Omega=\left\{x^{\prime} \in B(0,1) ;-f\left(x^{\prime}\right)<x_{N}<f\left(x^{\prime}\right)\right\}$, where $f: B(0,1) \rightarrow[1, \infty)$ is a smooth function. We test $\lambda_{2}^{H}$ with $v(x)=x_{N}|x|$. It is clear that $\int_{\Omega} \frac{v}{|x|^{2}} d x=0$. Moreover, we have

$$
\int_{\Omega}\left|\nabla\left(x_{N}|x|\right)\right|^{2} d x=\int_{\Omega}\left(4 x_{N}^{2}+\left|x^{\prime}\right|^{2}\right) d x=\frac{8}{3} \int_{B(0,1)} f\left(x^{\prime}\right)^{3} d x^{\prime}+2 \int_{B(0,1)}\left|x^{\prime}\right|^{2} f\left(x^{\prime}\right) d x^{\prime}
$$

and

Hence

$$
\int_{\Omega} \frac{v^{2}}{|x|^{2}} d x=\int_{\Omega} x_{N}^{2} d x=\frac{2}{3} \int_{B(0,1)} f\left(x^{\prime}\right)^{3} d x^{\prime}
$$

$$
\lambda_{2}^{H} \leq \frac{2 \int_{B(0,1)}\left|x^{\prime}\right|^{2} f\left(x^{\prime}\right) d x^{\prime}+\frac{8}{3} \int_{B(0,1)} f\left(x^{\prime}\right)^{3} d x^{\prime}}{\frac{2}{3} \int_{B(0,1)} f\left(x^{\prime}\right)^{3} d x^{\prime}}
$$

Since $f\left(x^{\prime}\right) \geq 1$ on $B(0,1)$, we derive from this estimate that $\lambda_{2}^{H} \leq 7$ for every $N$. Condition (3.1) is satisfied for $N \geq 10$. In the Appendix we give a slightly better estimate of $\lambda_{2}^{H}$. 


\section{Asymptotic behaviour of the second eigenfunction around 0}

To examine the behaviour around 0 of the second eigenfunction of (1.1) we use some ideas from [15], [18]. We denote by $S=S(\Omega)$ the best Sobolev constant for the embedding of $W^{1,2}(\Omega)$ into $L^{2^{*}}(\Omega)$, that is,

$$
S=\inf _{u \in W^{1,2}(\Omega)-\{0\}} \frac{\int_{\Omega}\left(|\nabla u|^{2}+u^{2}\right) d x}{\left(\int_{\Omega}|u|^{2^{*}} d x\right)^{\frac{2}{2^{*}}}} .
$$

We only consider the case $0 \in \Omega$.

Proposition 5.1 Suppose that $\lambda_{2}^{H}<\Lambda_{N}$. Then there exists $\delta_{\circ} \in(0,1)$ such that the eigenfunction $\varphi_{2}$ (constructed in Theorem 3.1) belongs to $L^{\left(1+\delta_{\circ}\right) 2^{*}}(\Omega)$.

Proof To simplify notation we set $\lambda=\lambda_{2}^{H}$ and $u=\varphi_{2}$. Let $\phi=u \min (|u|, L)^{p-2}=u u_{L}^{p-2}$, where $p>2, L>0$. Testing equation (1.1) we obtain

$$
\int_{\Omega}|\nabla u|^{2} u_{L}^{p-2} d x+(p-2) \int_{\Omega} \nabla u \nabla u_{L} u_{L}^{p-2} d x=\lambda \int_{\Omega} \frac{u^{2} u_{L}^{p-2}}{|x|^{2}} d x .
$$

On the other hand we have

$$
\begin{aligned}
\int_{\Omega}\left|\nabla\left(u u_{L}^{\frac{p}{2}-1}\right)\right|^{2} d x & =\int_{\Omega}|\nabla u|^{2} u_{L}^{p-2} d x+\frac{(p-2)^{2}}{4} \int_{\Omega}\left|\nabla u_{L}\right|^{2} u_{L}^{p-2} d x \\
& +(p-2) \int_{\Omega} \nabla u \nabla u_{L} u_{L}^{p-2} d x \\
& =\int_{\Omega}|\nabla u|^{2} u_{L}^{p-2} d x+\frac{p^{2}-4}{4} \int_{\Omega}\left|\nabla u_{L}\right|^{2} u_{L}^{p-2} d x .
\end{aligned}
$$

It then follows from (5.1) that

$$
\int_{\Omega}|\nabla u|^{2} u_{L}^{p-2} d x+\frac{p^{2}-4}{4} \int_{\Omega} \nabla u \nabla u_{L} u_{L}^{p-2} d x \leq \frac{\lambda(p+2)}{4} \int_{\Omega} \frac{u^{2} u_{L}^{p-2}}{|x|^{2}} d x .
$$

Hence

$$
\int_{\Omega}\left|\nabla\left(u u_{L}^{\frac{p}{2}-1}\right)\right|^{2} d x \leq \frac{\lambda(p+2)}{4} \int_{\Omega} \frac{u^{2} u_{L}^{p-2}}{|x|^{2}} d x .
$$

We now apply inequality (1.3) to $u u_{L}^{\frac{p}{2}-1}$. For a given $\epsilon>0$ we can find a constant $A=A(\epsilon, \Omega)>0$ such that

$$
\int_{\Omega}\left|\nabla\left(u u_{L}^{\frac{p}{2}-1}\right)\right|^{2} d x \leq \frac{\lambda(p+2)}{4}\left(\frac{1}{\Lambda_{N}}+\epsilon\right) \int_{\Omega}\left|\nabla\left(u u_{L}^{\frac{p}{2}-1}\right)\right|^{2} d x+\frac{\lambda A(p+2)}{4} \int_{\Omega} u^{2} u_{L}^{p-2} d x
$$


We now take $p=2+\delta$ with $0<\delta<1$ chosen so that

$$
\frac{\lambda}{\Lambda_{N}}\left(1+\frac{\delta}{4}\right)<1
$$

Next we choose $\epsilon$ small enough so that

$$
\frac{\lambda(p+2)}{4}\left(\frac{1}{\Lambda_{N}}+\epsilon\right)=\left(1+\frac{\delta}{4}\right)\left(\frac{\lambda}{\Lambda_{N}}+\lambda \epsilon\right)<1 .
$$

With these choices of $\delta$ and $\epsilon$ we derive from (5.3) that

$$
S\left(1-\frac{\lambda(p+2)}{4}\left(\frac{1}{\Lambda_{N}}+\epsilon\right)\right)\left(\int_{\Omega}\left|\left(u u_{L}^{\frac{p}{2}-1}\right)\right|^{2^{*}} d x\right)^{\frac{2}{2^{*}}} \leq\left(\frac{A \lambda(p+2)}{4}+1\right) \int_{\Omega} u^{2} u_{L}^{p-2} d x .
$$

Letting $L \rightarrow \infty$ the result follows with $\delta_{\circ}=\frac{\delta}{2}$.

Theorem 5.2 Suppose that $0 \in \Omega$ and $\lambda_{2}^{H}<\lambda_{N}$. Then there exists a ball $B\left(0, \rho_{\circ}\right) \subset \Omega$ such that

$$
\left|\varphi_{2}(x)\right| \leq C|x|^{-\left(\sqrt{\Lambda_{N}}-\sqrt{\Lambda_{N}-\lambda_{2}^{H}}\right)} \text { for } x \in B\left(0, \rho_{\circ}\right)-\{0\},
$$

where $C>0$ is a constant and $\varphi_{2}$ is an eigenfunction from Theorem 3.1.

Proof We use again the notation $\lambda=\lambda_{2}^{H}$ and we set $u=\varphi_{2}$. We put

$$
v(x)=|x|^{\left(\sqrt{\Lambda_{N}}-\sqrt{\Lambda_{N}-\lambda_{2}^{H}}\right)} u(x) .
$$

It is clear that $v \in W^{1,2}\left(\Omega,|x|^{-2\left(\sqrt{\Lambda_{N}}-\sqrt{\Lambda_{N}-\lambda_{2}^{H}}\right)} d x\right)$. By straightforward calculations we check that

$$
\operatorname{div}\left(|x|^{-2\left(\sqrt{\Lambda_{N}}-\sqrt{\Lambda_{N}-\lambda_{2}^{H}}\right)} \nabla v\right)=0 \text { in } \Omega
$$

Let $0<r<\rho$ and $B(0, \rho) \subset \Omega$. We put $\phi=\eta^{2} v v_{l}^{2(s-1)}$, where $l, s>1, v_{l}=\min (|v|, l)$ and $\eta$ is a $C^{1}$-function such that $\eta=1$ on $B(0, r), \eta=0$ on $\Omega-B(0, \rho)$ and $|\nabla \eta| \leq \frac{4}{\rho-r}$ on $\Omega$. Testing (5.4) with $\phi$ we obtain

$$
\int_{\Omega}|x|^{-2\left(\sqrt{\Lambda_{N}}-\sqrt{\Lambda_{N}-\lambda_{2}^{H}}\right)}\left(2 \eta v v_{l}^{2(s-1)} \nabla \eta \nabla v+\eta^{2} v_{l}^{2(s-1)}|\nabla v|^{2}+2(s-1) \eta^{2} v_{l}^{2(s-1)}\left|\nabla v_{l}\right|^{2}\right) d x=0 .
$$

For every $\epsilon>0$ there exists $C(\epsilon)>0$ such that

$$
\begin{array}{r}
2 \int_{\Omega}|x|^{-2\left(\sqrt{\Lambda_{N}}-\sqrt{\Lambda_{N}-\lambda_{2}^{H}}\right)} \eta v v_{l}^{2(s-1)} \nabla \eta \nabla v d x \leq \epsilon \int_{\Omega}|x|^{-2\left(\sqrt{\Lambda_{N}}-\sqrt{\Lambda_{N}-\lambda_{2}^{H}}\right)} \eta^{2} v_{l}^{2(s-1)}|\nabla v|^{2} d x \\
+C(\epsilon) \int_{\Omega}|x|^{-2\left(\sqrt{\Lambda_{N}}-\sqrt{\Lambda_{N}-\lambda_{2}^{H}}\right)}|\nabla \eta|^{2} v^{2} v_{l}^{2(s-1)} d x .
\end{array}
$$


Taking $\epsilon=\frac{1}{2}$ we derive from (5.5) that

$$
\begin{array}{r}
\int_{\Omega}|x|^{-2\left(\sqrt{\Lambda_{N}}-\sqrt{\Lambda_{N}-\lambda_{2}^{H}}\right)}\left(\eta^{2} v_{l}^{2(s-1)}|\nabla v|^{2}+2(s-1) \eta^{2} v_{l}^{2(s-1)}\left|\nabla v_{l}\right|^{2}\right) d x \\
\leq C \int_{\Omega}|x|^{-2\left(\sqrt{\Lambda_{N}}-\sqrt{\Lambda_{N}-\lambda_{2}^{H}}\right)}|\nabla \eta|^{2} v^{2} v_{l}^{2(s-1)} d x .
\end{array}
$$

In the next step we use the Caffarelli - Kohn - Nirenberg inequality [12]:

$$
\left(\int_{\Omega}|x|^{-b p}|w|^{p} d x\right)^{\frac{2}{p}} \leq C_{a, b} \int_{\Omega}|x|^{-2 a}|\nabla w|^{2} d x
$$

for every $w \in W_{\circ}^{1,2}\left(\Omega,|x|^{-2 a} d x\right)$, where $-\infty<a<\frac{N-2}{2}, a \leq b \leq a+1, p=\frac{2 N}{N-2+2(b-a)}$ and $C_{a, b}>0$ is a constant depending on $a$ and $b$. We choose $a=b=\sqrt{\Lambda_{N}}-\sqrt{\Lambda_{N}-\lambda_{2}^{H}}<\frac{N-2}{2}$. In this case we have $p=2^{*}$. We then deduce from (5.6) and (5.7) with $w=\eta v v_{l}^{s-1}$ that

$$
\begin{aligned}
& \left(\int_{\Omega}|x|^{-2^{*}\left(\sqrt{\Lambda_{N}}-\sqrt{\Lambda_{N}-\lambda_{2}^{H}}\right)}\left|\eta v v_{l}^{s-1}\right|^{2^{*}} d x\right)^{\frac{2}{2^{*}}} \\
& \leq \quad C_{a, a} \int_{\Omega}|x|^{-2\left(\sqrt{\Lambda_{N}}-\sqrt{\Lambda_{N}-\lambda_{2}^{H}}\right)}\left|\nabla\left(\eta v v_{l}^{s-1}\right)\right|^{2} d x \\
& \leq \quad 2 C_{a, a} \int_{\Omega}|x|^{-2\left(\sqrt{\Lambda_{N}}-\sqrt{\Lambda_{N}-\lambda_{2}^{H}}\right)}\left(|\nabla \eta|^{2} v^{2} v_{l}^{2(s-1)}+\eta^{2} v_{l}^{2(s-1)}|\nabla v|^{2}\right. \\
& \left.\quad+(s-1)^{2} \eta^{2} v_{l}^{2(s-1)}\left|\nabla v_{l}\right|^{2}\right) d x \\
& \leq C s \int_{\Omega}|x|^{-2\left(\sqrt{\Lambda_{N}}-\sqrt{\Lambda_{N}-\lambda_{2}^{H}}\right)}|\nabla \eta|^{2} v^{2} v_{l}^{2(s-1)} d x .
\end{aligned}
$$

Since

$$
\int_{\Omega}|x|^{-2^{*}\left(\sqrt{\Lambda_{N}}-\sqrt{\Lambda_{N}-\lambda_{2}^{H}}\right)}|\eta|^{2^{*}} v^{2} v_{l}^{2^{*} s-2} d x \leq \int_{\Omega}|x|^{-2^{*}\left(\sqrt{\Lambda_{N}}-\sqrt{\Lambda_{N}-\lambda_{2}^{H}}\right)}\left|\eta v v_{l}^{s-1}\right|^{2^{*}} d x
$$

we can rewrite (5.8) as

$$
\left(\int_{\Omega}|x|^{-2^{*}\left(\sqrt{\Lambda_{N}}-\sqrt{\Lambda_{N}-\lambda_{2}^{H}}\right)}|\eta|^{2^{*}} v^{2} v_{l}^{2^{*} s-2} d x\right)^{\frac{2}{2^{*}}} \leq C s \int_{\Omega}|x|^{-2^{*}\left(\sqrt{\Lambda_{N}}-\sqrt{\Lambda_{N}-\lambda_{2}^{H}}\right)}|\nabla \eta|^{2} v^{2} v_{l}^{2(s-1)} d x .
$$

Due to the properties of the function $\eta$ the above inequality becomes

$$
\left(\int_{B(0, r)}|x|^{-2^{*}\left(\sqrt{\Lambda_{N}}-\sqrt{\Lambda_{N}-\lambda_{2}^{H}}\right)} v^{2} v_{l}^{2^{*} s-2} d x\right)^{\frac{2}{2^{*}}} \leq \frac{C s}{(\rho-r)^{2}} \int_{B(0, \rho)}|x|^{-2^{*}\left(\sqrt{\Lambda_{N}}-\sqrt{\Lambda_{N}-\lambda_{2}^{H}}\right)} v^{2} v_{l}^{2 s-2} d x .
$$

We now choose $\frac{N}{N-2}<s^{*}<\left(1+\delta_{\circ}\right) \frac{N}{N-2}$ and define the sequence

$$
s_{j}=s^{*}\left(\frac{2^{*}}{2}\right)^{j}, j=0,1,2, \ldots
$$


Letting $s=s_{j}$ in (5.9) we obtain

$$
\begin{aligned}
& \left(\int_{B(0, r)}|x|^{-2^{*}\left(\sqrt{\Lambda_{N}}-\sqrt{\Lambda_{N}-\lambda_{2}^{H}}\right)} v^{2} v_{l}^{2^{*} s_{j+1}-2} d x\right)^{\frac{1}{2 s_{j+1}}} \\
\leq & \left(\frac{C s_{j}}{(\rho-r)^{2}}\right)^{\frac{1}{2 s_{j}}}\left(\int_{B(0, \rho)}|x|^{-2^{*}\left(\sqrt{\Lambda_{N}}-\sqrt{\Lambda_{N}-\lambda_{2}^{H}}\right)} v^{2} v_{l}^{2 s_{j}-2} d x\right)^{\frac{1}{2 s_{j}}} .
\end{aligned}
$$

We put $r_{j}=\rho_{\circ}\left(1+\rho_{\circ}^{j}\right), j=0,1,2, \ldots$ with $\rho_{\circ}>0$ small so that $\overline{B\left(0,2 \rho_{\circ}\right)} \subset \Omega$. Substituting in the above inequality $\rho=r_{j}$ and $r=r_{j+1}$ we obtain

$$
\begin{aligned}
& \left(\int_{B\left(0, r_{j+1}\right)}|x|^{-2^{*}\left(\sqrt{\Lambda_{N}}-\sqrt{\Lambda_{N}-\lambda_{2}^{H}}\right)} v^{2} v_{l}^{2^{*} s_{j+1}-2} d x\right)^{\frac{1}{2 s_{j+1}}} \\
& \leq\left(\frac{C s_{j}}{\left(\rho_{\circ}-\rho_{\circ}^{2}\right)^{2} \rho_{\circ}^{2 j}}\right)^{\frac{1}{2 s_{j}}}\left(\int_{B\left(0, r_{j}\right)}|x|^{-2^{*}\left(\sqrt{\Lambda_{N}}-\sqrt{\Lambda_{N}-\lambda_{2}^{H}}\right)} v^{2} v_{l}^{2 s_{j}-2} d x\right)^{\frac{1}{2 s_{j}}} \text {. }
\end{aligned}
$$

Iterating we obtain

$$
\begin{aligned}
& \left(\int_{B\left(0, r_{j+1}\right)}|x|^{-2^{*}\left(\sqrt{\Lambda_{N}}-\sqrt{\Lambda_{N}-\lambda_{2}^{H}}\right)} v^{2} v_{l}^{2^{*} s_{j+1}-2} d x\right)^{\frac{1}{2 s_{j+1}}} \\
\leq & \left(\frac{C}{\rho_{\circ}-\rho_{\circ}^{2}}\right)^{\sum_{j=0}^{\infty} \frac{1}{s_{j}}} \rho_{\circ}^{-\sum_{j=0}^{\infty} \frac{1}{s_{j}}} \prod_{j=0}^{\infty} s_{j}^{\frac{1}{2 s_{j}}}\left(\int_{B\left(0, r_{j}\right)}|x|^{-2^{*}\left(\sqrt{\Lambda_{N}}-\sqrt{\Lambda_{N}-\lambda_{2}^{H}}\right)} v^{2} v_{l}^{2 s^{*}-2} d x\right)^{\frac{1}{2 s^{*}}} .
\end{aligned}
$$

We now notice that the infinite sums and the infinite product in the above inequality are finite. Since $2^{*}<2 s^{*}<\left(1+\delta_{\circ}\right) 2^{*}$ we get that

$$
\begin{aligned}
& \int_{B\left(0, r_{\circ}\right)}|x|^{-2^{*}\left(\sqrt{\Lambda_{N}}-\sqrt{\Lambda_{N}-\lambda_{2}^{H}}\right)} v^{2} v^{2 s^{*}-2} d x \\
\leq & \int_{B\left(0, r_{\circ}\right)}|x|^{\left(2 s^{*}-2^{*}\right)\left(\sqrt{\Lambda_{N}}-\sqrt{\Lambda_{N}-\lambda_{2}^{H}}\right)}|u|^{2 s^{*}} d x \\
\leq & d^{\left(2 s^{*}-2^{*}\right)\left(\sqrt{\Lambda_{N}}-\sqrt{\Lambda_{N}-\lambda_{2}^{H}}\right)} \int_{B\left(0, r_{\circ}\right)}|u|^{2 s *} d x<\infty .
\end{aligned}
$$

We now deduce from (5.11) and (5.12) that

$$
\begin{aligned}
\left\|v_{l}\right\|_{L^{2 s_{j+1}}\left(B\left(0, r_{\circ}\right)\right)} & \leq\left\|v_{l}\right\|_{L^{2 s_{j+1}}\left(B\left(0, r_{j+1}\right)\right)} \\
& \leq d^{\frac{2^{*}\left(\sqrt{\Lambda_{N}}-\sqrt{\Lambda_{N}-\lambda_{2}^{H}}\right)}{2 s_{j+1}}}\left(\int_{B\left(0, r_{j+1}\right)}|x|^{-2^{*}\left(\sqrt{\Lambda_{N}}-\sqrt{\Lambda_{N}-\lambda_{2}^{H}}\right)} v^{2} v_{l}^{2 s_{j+1}-2} d x\right)^{\frac{1}{2 s_{j+1}}} .
\end{aligned}
$$

Letting $l \rightarrow \infty$ and $s_{j} \rightarrow \infty$ the result easily follows. 


\section{The Robin boundary conditions}

The approach from Section 3 to the eigenvalue problem (1.1) can be extended to the eigenvalue problem with Robin boundary conditions

$$
\begin{cases}-\Delta u & =\mu \frac{1}{|x|^{2}} u \text { in } \Omega, \\ \frac{\partial u}{\partial \nu}+\beta u & =0 \text { on } \partial \Omega,\end{cases}
$$

where $\beta$ is a continuous nonnegative function on $\partial \Omega$ with $\beta \not \equiv 0$ on $\partial \Omega$. We assume that $0 \in \Omega$. However, we consider here the smallest eigenfunction

$$
\lambda_{1}^{R}=\inf _{u \in W^{1,2}(\Omega)-\{0\}} \frac{\int_{\Omega}|\nabla u|^{2} d x+\int_{\partial \Omega} \beta u^{2} d s}{\int_{\Omega} \frac{u^{2}}{|x|^{2}} d x} .
$$

First we show that $\lambda_{1}^{R}>0$. Arguing by contradiction assume $\lambda_{1}^{R}=0$. Then there exits a sequence $\left\{u_{n}\right\} \subset W^{1,2}(\Omega)$ such that $\int_{\Omega}\left|\nabla u_{n}\right|^{2} d x+\int_{\partial \Omega} \beta u_{n}^{2} d s \rightarrow 0$ as $n \rightarrow \infty$ and $\int_{\Omega} \frac{u_{n}^{2}}{|x|^{2}} d x=$ 1 for every $n$. Since $\left\{u_{n}\right\}$ is bounded in $W^{1,2}(\Omega)$ we may assume that $u_{n} \rightarrow u$ in $W^{1,2}(\Omega)$ and $u_{n} \rightarrow u$ in $L^{2}(\Omega)$ and $L^{2}(\partial \Omega)$. We now observe that $\int_{\Omega}\left|\nabla u_{n}\right|^{2} d x \rightarrow 0$, so $u=l$ (constant). Since $\beta \not \equiv 0, l=0$. Thus $u_{n} \rightarrow 0$ in $W^{1,2}(\Omega)$. This contradicts the fact that $\int_{\Omega} \frac{u_{n}^{2}}{|x|^{2}} d x=1$ for every $n$. Hence $\lambda_{1}^{R}>0$. Obviously, we have

$$
\lambda_{1}^{R} \leq \inf _{u \in W_{\circ}^{1,2}(\Omega)} \frac{\int_{\Omega}|\nabla u|^{2} d x}{\int_{\Omega} \frac{u^{2}}{|x|^{2}} d x}=\Lambda_{N} .
$$

We now recall the following inequality: there exists a constant $C=C(\Omega)>0$ such that

$$
\Lambda_{N} \int_{\Omega} \frac{u^{2}}{|x|^{2}} d x \leq \int_{\Omega}|\nabla u|^{2} d x+C \int_{\partial \Omega} u^{2} d s
$$

for every $u \in W^{1,2}(\Omega)$ (see Theorem 1.1 (inequality (1.4)) in [7]). Actually, this is a simplified version of inequality (1.4) in [7], which contains an extra positive term on the left hand side. Let $C$ be a constant from inequality (6.3). If $C \leq \beta(s)$ on $\partial \Omega$, then $\lambda_{1}^{R} \geq \Lambda_{N}$. Thus if $C \leq \beta(s)$ on $\partial \Omega$ then $\lambda_{1}^{R}=\Lambda_{N}$.

Theorem 6.1 Suppose that

$$
\lambda_{1}^{R}<\Lambda_{N}
$$

Then problem (6.2) admits a minimizer.

The proof is a minor modification of the proof of Theorem 3.1 and is omitted.

Using as a test function $u(x) \equiv 1$ on $\Omega$ we derive the following condition guaranteeing the validity of $(6.4)$ : if

$$
\int_{\partial \Omega} \beta(s) d s<\Lambda_{N} \int_{\Omega} \frac{d x}{|x|^{2}}
$$


then (6.4) holds. If $\Omega=B(0, R)$, and $B=\max _{s \in \partial \Omega} \beta(s)$, then

$$
\frac{\int_{\partial \Omega} \beta(s) d s}{\int_{\Omega} \frac{d x}{|x|^{2}}} \leq B R(N-2)
$$

and (6.5) becomes

$$
R<\frac{N-2}{4 B}
$$

Proposition 6.2 Suppose that (6.4) holds. Then the first eigenvalue $\lambda_{1}^{R}$ is simple and the corresponding eigenfunction does not change sign on $\Omega-\{0\}$.

Proof If a function $u \in W^{1,2}(\Omega)$ is a minimizer for $\lambda_{1}^{R}$, then $|u|$ is also a minimizer for $\lambda_{1}^{R}$. Therefore we can assume that $u$ is nonnegative on $\Omega-\{0\}$. The fact that $u>0$ on $\Omega-\{0\}$ follows from the Harnack inequality (see Theorem 8.20 in [17]). To prove that $\lambda_{1}^{R}$ is simple we follow some ideas from [9] and [24]. We use the Picone identity. Let

$$
L(u, v)=|\nabla u|^{2}+\frac{u^{2}}{v^{2}}|\nabla v|^{2}-2 \frac{u}{v} \nabla u \nabla v
$$

and

$$
R(u, v)=|\nabla u|^{2}-\nabla v \nabla\left(\frac{u^{2}}{v}\right) .
$$

If $u, v \in C^{1}(\Omega-\{0\}) \cap W^{1,2}(\Omega)$, with $u \geq 0, v>0$, then $R(u, v)=L(u, v) \geq 0$. Moreover, $R(u, v)=L(u, v)=0$ if and only if $u=k v$ for some $k \in \mathbb{R}$. Suppose that $\phi_{1}$ and $v_{1}$ are positive eigenfunctions corresponding to $\lambda_{1}^{R}$. We choose a sequence $\psi_{n} \in C(\Omega) \cap W^{1,2}(\Omega)$ such that $\psi_{n} \rightarrow \phi_{1}$ in $W^{1,2}(\Omega)$. We then have

$$
\begin{aligned}
0 & =\int_{\Omega}\left|\nabla \phi_{1}\right|^{2} d x+\int_{\partial \Omega} \beta \phi_{1}^{2} d s-\lambda_{1}^{R} \int_{\Omega} \frac{\phi_{1}^{2}}{|x|^{2}} d x \\
& =\lim _{n \rightarrow \infty}\left[\int_{\Omega}\left|\nabla \psi_{n}\right|^{2} d x+\int_{\partial \Omega} \beta \psi_{n}^{2} d s-\lambda_{1}^{R} \int_{\Omega} \frac{\psi_{n}^{2}}{|x|^{2}} d x\right] .
\end{aligned}
$$

Testing the equation for $v_{1}$ with $v=\frac{\psi_{n}^{2}}{v_{1}+\frac{1}{n}}$ we get

$$
-\int_{\partial \Omega} \beta \psi_{n}^{2} \frac{v_{1}}{\left(v_{1}+\frac{1}{n}\right)} d s+\lambda_{1}^{R} \int_{\Omega} \frac{v_{1}}{\left(v_{1}+\frac{1}{n}\right)} \frac{\psi_{n}^{2}}{|x|^{2}} d x=\int_{\Omega} \nabla v_{1} \nabla\left(\frac{\psi_{n}^{2}}{v_{1}+\frac{1}{n}}\right) d x
$$

Combining this with (6.6) we derive

$$
0=\lim _{n \rightarrow \infty} \int_{\Omega}\left(\left|\nabla \psi_{n}\right|^{2}-\nabla v_{1} \nabla\left(\frac{\psi_{n}^{2}}{v_{1}+\frac{1}{n}}\right)\right) d x=\lim _{n \rightarrow \infty} \int_{\Omega} L\left(\psi_{n}, v_{1}\right) d x=\int_{\Omega} L\left(\phi_{1}, v_{1}\right) d x .
$$

Thus $\phi_{1}=c v_{1}$ for some $c \in \mathbb{R}$.

The asymptotic estimates from Theorem 5.2 continue to hold for the first eigenfunction of problem (6.1). 
Proposition 6.3 Suppose that (6.4) holds and let $v$ be a minimizer of problem (6.2). Then there exist positive constants $C_{1}, C_{2}$ and $\rho_{\circ}$ such that $B\left(0, \rho_{\circ}\right) \subset \Omega$ and

$$
C_{1}|x|^{-\left(\sqrt{\Lambda_{N}}-\sqrt{\Lambda_{N}-\lambda_{1}^{R}}\right)} \leq v(x) \leq C_{2}|x|^{-\left(\sqrt{\Lambda_{N}}-\sqrt{\Lambda_{N}-\lambda_{1}^{R}}\right)}
$$

for every $x \in B\left(0, \rho_{\circ}\right)-\{0\}$.

Proof First we observe that Proposition 5.1 remains true for $v$. Hence we can repeat the proof of Theorem 5.2 to obtain the upper bound. The lower bound follows from Proposition 2.2 in $[15]$.

We now consider the Robin problem (6.1) with a non-positive coefficient $\beta$ with $\beta \not \equiv 0$. We rewrite this problem as

$$
\begin{cases}-\Delta u & =\mu \frac{1}{|x|^{2}} u \text { in } \Omega \\ \frac{\partial u}{\partial \nu}-\beta u & =0 \text { on } \partial \Omega\end{cases}
$$

where $\beta$ is a continuous non-negative function on $\partial \Omega$ with $\beta \not \equiv 0$. We put

$$
\lambda_{1}^{-R}=\inf _{u \in W^{1,2}(\Omega)-\{0\}} \frac{\int_{\Omega}|\nabla u|^{2} d x-\int_{\partial \Omega} \beta u^{2} d s}{\int_{\Omega} \frac{u^{2}}{|x|^{2}} d x} .
$$

Theorem 6.4 Suppose that $\beta$ is a continuous non-negative function on $\partial \Omega$ with $\beta \not \equiv 0$. Then problem (6.8) admits a minimizer.

Proof We commence by showing that $-\infty<\lambda_{1}^{-R}<0$. Testing $\lambda_{1}^{-R}$ with $u \equiv 1$ we get $\lambda_{1}^{-R}<0$. To show that $\lambda_{1}^{-R}$ is finite we recall the following version of the trace inequality in $W^{1,2}(\Omega)$ : for every $\epsilon>0$ there exists a constant $C(\epsilon)>0$ such that

$$
\int_{\partial \Omega} u^{2} d s \leq \epsilon \int_{\Omega}|\nabla u|^{2} d x+C(\epsilon) \int_{\Omega} u^{2} d x
$$

for every $u \in W^{1,2}(\Omega)$. We choose $\epsilon>0$ so that $\epsilon\|\beta\|_{\infty}<1$. With this choice of $\epsilon$ we obtain the following estimate

$$
\int_{\Omega}|\nabla u|^{2} d x-\int_{\partial \Omega} \beta u^{2} d s \geq\left(1-\epsilon\|\beta\|_{\infty}\right) \int_{\Omega}|\nabla u|^{2} d x-\|\beta\|_{\infty} C(\epsilon) \int_{\Omega} u^{2} d x .
$$

We may assume that $\int_{\Omega} \frac{u^{2}}{|x|^{2}} d x=1$. Hence $1=\int_{\Omega} \frac{u^{2}}{|x|^{2}} d x \geq \frac{1}{d^{2}} \int_{\Omega} u^{2} d x$. We then derive the following estimate

$$
\int_{\Omega}|\nabla u|^{2} d x-\int_{\partial \Omega} \beta u^{2} d s \geq-d^{2}\|\beta\|_{\infty} C(\epsilon) .
$$

This argument also shows that every minimizing sequence for $\lambda_{1}^{-R}$ must be bounded in $W^{1,2}(\Omega)$. Let $\left\{u_{n}\right\} \subset W^{1,2}(\Omega)$ be a minimizing sequence for $\lambda_{1}^{-R}$ such that $\int_{\Omega} \frac{u_{n}^{2}}{|x|^{2}} d x=1$ for every $n$. We may assume that $u_{n} \rightarrow u$ in $W^{1,2}(\Omega)$ and $L^{2}\left(\Omega, \frac{1}{|x|^{2}}\right)$ and $u_{n} \rightarrow u$ in $L^{2}(\Omega)$ 
and $L^{2}(\partial \Omega)$. We claim that $u \not \equiv 0$. In the contrary case by the P.L. Lions concentration - compactness principle we get (see the proof of Theorem 3.1) $0>\lambda_{1}^{-R} \geq \mu_{\circ}$ which is impossible. To complete the proof we show that $\lim _{n \rightarrow \infty} \int_{\Omega}\left|\nabla u_{n}-\nabla u\right|^{2} d x=0$. Arguing by contradiction assume $\lim _{n \rightarrow \infty} \int_{\Omega}\left|\nabla u_{n}-\nabla u\right|^{2} d x=c>0$. Since $\left\{u_{n}\right\}$ is a minimizing sequence for $\lambda_{1}^{-R}$ we obtain

$$
\begin{aligned}
\lambda_{1}^{-R} & =\int_{\Omega}\left|\nabla u_{n}\right|^{2} d x-\int_{\partial \Omega} \beta u_{n}^{2} d s+o(1)=c+\int_{\Omega}|\nabla u|^{2} d x-\int_{\partial \Omega} \beta u^{2} d s+o(1) \\
& \geq c+\lambda_{1}^{-R} \int_{\Omega} \frac{u^{2}}{|x|^{2}} d x+o(1) .
\end{aligned}
$$

Hence

$$
c \leq \lambda_{1}^{-R}\left(1-\int_{\Omega} \frac{u^{2}}{|x|^{2}} d x\right) \leq 0
$$

and we have arrived at a contradiction.

We close this section with remarks on the Robin problem with the coefficient $\beta$ changing sign on $\partial \Omega$, that is, we assume $\beta^{+} \not \equiv 0$ and $\beta^{-} \not \equiv 0$ on $\partial \Omega$. We distinguish two cases: (I) $\int_{\partial \Omega} \beta d x \leq 0$ and (II) $\int_{\partial \Omega} \beta d s>0$. Using the trace inequality (6.9) we can show that $\lambda_{1}^{R}$ is finite.

Case (I) In this case $\lambda_{1}^{R}<0$. This is obvious if $\int_{\Omega} \beta d x<0$. If $\int_{\partial \Omega} \beta d s=0$, we test $\lambda_{1}^{R}$ with a function $u(x)=v(x)+t$, where $t>0$ is a constant and $v \in C^{1}(\bar{\Omega}), v \geq 0$ on $\Omega$, $\int_{\partial \Omega} \beta^{-} v d s>0$ and $\operatorname{supp} v \cap \operatorname{supp} \beta^{+}=\emptyset$. Thus

$$
\begin{aligned}
\int_{\Omega}|\nabla u|^{2} d x+\int_{\partial \Omega} \beta u^{2} d s & =\int_{\Omega}|\nabla v|^{2} d x+\int_{\partial \Omega} \beta v^{2} d s+2 t \int_{\partial \Omega} \beta v d s+t^{2} \int_{\partial \Omega} \beta d s \\
& =\int_{\Omega}|\nabla v|^{2} d x-\int_{\partial \Omega} \beta^{-} v^{2} d s-2 t \int_{\partial \Omega} \beta^{-} v d s<0
\end{aligned}
$$

provided $t$ is sufficiently large. Repeating the proof of Theorem 6.4 we obtain the existence of a minimizer for $\lambda_{1}^{R}$.

Case (II) In this case

$$
\lambda_{1}^{R} \leq \frac{\int_{\partial \Omega} \beta d s}{\int_{\Omega} \frac{d x}{|x|^{2}}} .
$$

To obtain the existence of a minimizer for $\lambda_{1}^{R}$ we impose the following condition

$$
\int_{\partial \Omega} \beta d s<\Lambda_{N} \int_{\Omega} \frac{d x}{|x|^{2}}
$$

Obviously this condition is not needed if it occurs that $\lambda_{1}^{R} \leq 0$. 


\section{Open problems}

Listed below are some open problems.

1) Do there exist second eigenfunctions in balls with dimensions less than or equal to 6 ?

2) Do there exist other eigenvalues below the Hardy constant $\Lambda_{N}$ and possibly above $\Lambda_{N}$ ?

3) Find conditions for the mixed Dirichlet - Neumann problem guaranteeing that the corresponding minimum of the Rayleigh quotient is strictly less than $\Lambda_{N}$ and is attained.

\section{Appendix}

Let $\Omega$ be a domain from example 7 in Section 4 . We test $\lambda_{2}^{H}$ with $v(x)=x_{N}^{\frac{2 k+1}{2 k+3}}|x|$, where $k \geq 1$ is an integer. We have

$$
\nabla v=\left(x_{N}^{\frac{2 k+1}{2 k+3}} \frac{x_{1}}{|x|}, \ldots, x_{N}^{\frac{2 k+1}{2 k+3}} \frac{x_{N-1}}{|x|}, \frac{2 k+1}{2 k+3} x_{N}^{\frac{-2}{2 k+3}}|x|+x_{N}^{\frac{2 k+1}{2 k+3}} \frac{x_{N}}{|x|}\right) .
$$

Thus

$$
\begin{aligned}
\int_{\Omega}|\nabla v|^{2} d x & =\int_{\Omega}\left(x_{N}^{\frac{4 k+2}{2 k+3}}+\left(\frac{2 k+1}{2 k+3}\right)^{2} x_{N}^{-\frac{4}{2 k+3}}|x|^{2}+\frac{4 k+2}{2 k+3} x_{N}^{\frac{4 k+2}{2 k+3}}\right) d x \\
& =\int_{\Omega}\left(x_{N}^{\frac{4 k+2}{2 k+3}}+\left(\frac{2 k+1}{2 k+3}\right)^{2} x_{N}^{\frac{4 k+2}{2 k+3}}+\left(\frac{2 k+1}{2 k+3}\right)^{2} x_{N}^{-\frac{4}{2 k+3}}\left|x^{\prime}\right|^{2}+\frac{4 k+2}{2 k+3} x_{N}^{\frac{4 k+2}{2 k+3}}\right) d x \\
& =2\left(\frac{2 k+3}{6 k+5} \int_{\left|x^{\prime}\right| \leq 1} f\left(x^{\prime}\right)^{\frac{6 k+5}{2 k+3}} d x^{\prime}+\left(\frac{2 k+1}{2 k+3}\right)^{2} \frac{2 k+3}{6 k+5} \int_{\left|x^{\prime}\right| \leq 1} f\left(x^{\prime}\right)^{\frac{6 k+5}{2 k+3}} d x^{\prime}\right. \\
& \left.+\left(\frac{2 k+1}{2 k+3}\right)^{2} \frac{2 k+3}{2 k-1} \int_{\left|x^{\prime}\right| \leq 1}\left|x^{\prime}\right|^{2} f\left(x^{\prime}\right)^{\frac{2 k-1}{2 k+1}} d x^{\prime}+\frac{4 k+2}{6 k+5} \int_{\left|x^{\prime}\right| \leq 1} f\left(x^{\prime}\right)^{\frac{6 k+5}{2 k+3}} d x^{\prime}\right) \\
& \leq 2\left(\frac{2 k+3}{6 k+5}+\left(\frac{2 k+1}{2 k+3}\right)^{2} \frac{2 k+3}{6 k+5}+\left(\frac{2 k+1}{2 k+3}\right)^{2} \frac{2 k+3}{2 k-1}+\frac{4 k+2}{6 k+5}\right) \\
& \times \int_{\left|x^{\prime}\right| \leq 1} f\left(x^{\prime}\right)^{\frac{6 k+5}{2 k+3}} d x^{\prime} .
\end{aligned}
$$

We also have

$$
\int_{\Omega} \frac{v^{2}}{|x|^{2}} d x \leq \int_{\Omega} x_{N}^{\frac{4 k+2}{2 k+3}} d x=2 \frac{2 k+3}{6 k+5} \int_{\Omega} f\left(x^{\prime}\right)^{\frac{6 k+5}{2 k+3}} d x^{\prime}
$$

Since $\int_{\Omega} \frac{v}{\left|x^{\prime}\right|^{2}} d x=0, v$ is a legitimate function to test $\lambda_{2}^{H}$ and we get

$$
\lambda_{2}^{H} \leq\left(\frac{2 k+3}{6 k+5}+\left(\frac{2 k+1}{2 k+3}\right)^{2} \frac{2 k+3}{6 k+5}+\left(\frac{2 k+1}{2 k+3}\right)^{2} \frac{2 k+3}{2 k-1}+\frac{4 k+2}{6 k+5}\right) \frac{6 k+5}{2 k+3} .
$$


The sequence on the right - hand side of this inequality tends to 7 as $k \rightarrow \infty$. However, the terms of this sequence are strictly less than 6 for $k=2,3,4,5$. For example for $k=2$ we have

$$
\lambda_{2}^{H} \leq 1+\frac{25}{49}+\frac{25}{49} \cdot \frac{17}{3}+\frac{10}{7}<5 \frac{122}{147}<6 .
$$

In particular, condition (3.1) is satisfied for $N \geq 7$.

\section{References}

[1] B. Abdellaoui and I. Peral, Existence and nonexistence results for quasilinear elliptic equations involving the p-Laplacian with a critical potential, Annali di Mat. Pura e Appl. (2003), 182(3), 247-270.

[2] B. Abdellaoui and I. Peral, Nonexistence results for quasilinear elliptic equations related to Caffarelli-Kohn-Nirenberg inequalities,Comm. in Pure Appl. Anal. (2003) 2(4), 539566.

[3] B. Abdellaoui, E. Colorado and I. Peral, Some remarks on elliptic equations with singular potentials and mixed boundary conditions, Advanced Nolinear Stuadies (A Volume Dedicated to A. Ambrossetti on occasin of his 60 birthday) (20004), 501-531.

[4] B. Abdellaoui, E. Colorado and I. Peral, Some improved Caffarelli-Kohn-Nirenberg inequalities Calc. Var. and PDE, (2005) 23, 327-345.

[5] B. Abdellaoui, E. Colorado and I. Peral, Effect of the boundary conditions in behavior of the optimal constant of some Caffarelli-Kohn-Nirenberg inequalities: Applications to critical nonlinear problems, Advances in Diff. Equations (2006) 11(6), 667-720.

[6] Adimurthi, Hardy Sobolev inequality in $H^{1}(\Omega)$ and its applications, Communications in Contemp. Maths (2002) 4, 409-439.

[7] Adimurthi and M.J. Esteban, An improved Hardy - Sobolev inequality in $W^{1, p}(\Omega)$ and its applications to Schrödinger operators, NoDEA (2005) 12, 243-263.

[8] G.A. Afrouzi and K.J. Brown, On principal eigenvalues for boundary value problems with indefinite weight and Robin boundary conditions, Proc. Am. Math. Soc.(1999), $127,125-130$.

[9] W. Allegretto and Y.X. Hang, A Picone identity for the p-Laplacian and applications, Nonlin. Anal. TMA (1998), 32, 819-830.

[10] H. Brezis and J.L. Vázquez, Blow - up solutions of some nonlinear elliptic problems, Revista Mat. Univ. Complut. de Madrid, 10(2) (1997), 443-469.

[11] K.J. Brown and S.S. Lin, On the existence of positive eigenfunctions for an eigenvalue problem with indefinite weight function, J. Math. Anal. Appl. (1980), 75, 112-120. 
[12] L. Caffarelli, R. Kohn and L. Nirenberg, First order interpolation inequalities with weights, Compositio Math., 53 (1984), 259-275.

[13] Daomin Cao and J. Chabrowski, Critical Neumann problem with competing Hardy potentials, Rev. Mat. Complut. 20 (2007), no. 2, 309-338.

[14] J. Chabrowski, On the nonlinear Neumann problem involving the critical Sobolev exponent and Hardy potential, Rev. Mat. Complut. (2004) 17; Núm. 1, 195-227.

[15] Jianqino Chen, Exact local behavior of positive solutions for a semilinear elliptic equations with Hardy term, Proc. Am. Math. Soc. (2004) 132 (11), 3225-3229.

[16] E. Colorado and I. Peral, Eigenvalues and bifurcation for elliptic equations related to Caffarelli-Kohn-Nirenberg inequalities with mixed Dirichlet- Neumann boundary conditions, Topological Methods in Nonlin. Anal. (2004) 23, 239-273.

[17] D. Gilbarg and N.S. Trudinger, Elliptic Partial Differential Equations of Second Order, Springer - Verlag Berlin Heidelberg 1983 (second edition).

[18] Pigong Han, Asymptotic behavior of solutions to semilinear elliptic equations with Hardy potential, Proc. Amer. Math. Soc. 135 (2007), 365-372

[19] Pigong Han and Zhaoxia Liu, Solutions to nonlinear Neumann problems with inverse square potential, Calc. Var. (2007) 30, 315-352.

[20] An Lê, Eigenvalue problems for the p-Laplacian, Nonlinear Analysis, (2006) 64 (5), 1057-1099.

[21] Elliot H. Lieb and Michael Loss, Analysis, AMS, vol. 14 (2001) (second edition).

[22] E.H. Lieb, R. Seiringer and J. Yngvason, Poincaré inequality in punctured domains, Annals of Maths (2003) 158, 1067-1080.

[23] P.L. Lions, The concentration - compactness principle in calculus of variations. The limit case I, Rev. Mat. Iberoamericana (1985), 145-201.

[24] K. Sreenadh, On the eigenvalue problem for the Hardy - Sobolev operator with indefinite weights, Electronic J. Diff. Equations (2002) Vol. 2002, No.33, 1-12.

[25] Kenichiro Umezu, On eigenvalue problems with Robin type boundary conditions having indefinite coefficients, Appl. Analysis, (2006), 85(11), 1313-1325. 